\title{
Guest Researchers in Japan
}

\section{Thomas W. Ebbesen}

The opportunities for foreigners to do research in Japan are better than ever before. However, that does not mean that these opportunities cannot be further improved nor that it is any less challenging than in the past. A stay in Japan can be an enriching experience or a frustration, depending on the ability of the visitors and their hosts to understand the expectations of one another from the visit. Here I discuss some of the opportunities, then explain some difficulties arising from language barriers and cultural differences in trying to meet these expectations. Needless to say, the cultural gap is very big between the West and Japan. This gap often leads to misunderstandings and sometimes to serious problems. It is also the breeding ground for sensationalist views on the Japanese experience.

\section{Opportunities}

The situation for foreign scientists in Japan has significantly changed over the last 10 years. ${ }^{1-3}$ The number of foreign scientists is ever increasing, with many of the larger institutes, whether private or public, hosting a hundred or more within a given year. ${ }^{3}$ The number of foreign graduate students has also increased considerably and now stands at about 15,000. Working in Japan for either a month or 1-2 years is now common.

For U.S. scientists, funding may be provided from the Japanese host institutions or from organizations such as the National Science Foundation (NSF). The NSF-Japan program offers a variety of options ranging from a summer initiation-to-Japan program for graduate students to a sabbatical year for a professor. The NSF support is available for scientists going to either academic or industrial laboratories, offering considerable flexibility.

Host institutions in Japan can be loosely categorized into four types: universities, national laboratories, national projects, and industrial laboratories. The big national universities have opened up to foreign students in the last 10 years and the number of visiting professor positions has increased. Permanent positions are still very rare in national universities, but not in private universities, 1,2 Unfortunately universities are typically not as well equipped as other private or public institutions. There are exceptions such as the special institutes attached to the universities or certain privileged laboratories. National laboratories of various Ministries, other than the Ministry of Education, were relatively late in offering positions for foreigners but now offer real opportunities: postdoctoral positions, visiting, and permanent positions. The national laboratories are typically well equipped. Also, various national projects (ERATO, Frontier, etc.) provide good funding limited to a 5 or 10 year period. While many of the projects are supposed to be at the frontier of science, they are often very technology oriented. In any case, they offer the chance for foreign postdoctorates to be involved at the cutting edge of a certain domain.

\section{Since position openings are rarely announced publicly, one has to write directly to the person of interest.}

With about $80 \%$ of the Japanese R\&D expenditure coming from industry, industrial laboratories may offer serious alternatives to foreign scientists and engineers depending on their field of expertise. During the last 10 years, as industry has opened its doors for basic research, the number of foreigners has been increasing rapidly. Not all industry laboratories are equally open to foreigners, but larger companies with better laboratories tend to be the most open. ${ }^{3}$ They

Material Matters is a forum for expressing personal points of view on issues of interest to the materials community. mostly offer postdoctoral and visiting positions for senior scientists, but occasionally open permanent positions.

\section{Tips for Potential Visitors Making Arrangements}

Some scientists visit Japan out of curiosity to discover a new country and its culture, in which case the host institution and host group is not perhaps so critical. However, if the aim is to achieve scientific results, solid preparations must be made for the trip. This is especially important for postdoctorates who, upon their return to their home country, will have to show what they achieved during their stay in order to get a job. Simply having been in Japan and speaking a bit of the language will not benefit scientists during a job search outside of Japan; only results will. There are now so many foreigners who, having grown up in Japan, or graduated from Japanese universities, have more than a superficial understanding of the language and the culture.

For results, the best starting point is knowing a person or a laboratory for whose work one has serious interest and respect. Since position openings are rarely announced publicly, one has to write directly to the person of interest, or get an introduction through a common friend or a professor, or if possible try to meet contacts at a conference. If other foreigners have done well in the same place, it is a good sign. In institutes that have a large number of foreigners, the Japanese are used to their presence and they are treated like everyone else. This is the most comfortable situation. The Japanese hosts are more likely to know the expectations of their visitors and visitors can learn from other visiting researchers how to function and integrate.

Special treatment lavished on foreign researchers may seem nice in the beginning, but it generally leads to problems such as isolation or the ivory-tower treatment. The latter can be a problem particularly for senior scientists wishing to spend a sabbatical year doing experiments hands-on. A visitor might be wined and dined across Japan in a lecture tour organized by the host and given a large office, but never do an experiment. Why? Other researchers are too busy with their own functions in the host group and they might never have seen anyone of the visitor's rank do an experiment. ${ }^{4}$ So senior scientists spending a year in a Japanese laboratory must make their expectations very clear. A visitor planning to do experiments should confirm in advance an agreement of collaborative work with a scientist in the host 
group. Since group leaders do not typically do experiments, they will have to assign or convince junior staff or graduate students to work with the visiting researcher. Such collaboration is necessary since work procedures are done differently and many small barriers such as understanding instructions in Japanese in the computer must be overcome.

Preparing well for the visit is also important for another reason. It takes time to start a collaborative project or a research theme in Japan. A collaboration, even a short one, will require greetings, meetings, and discussion back and forth before starting. Access time to a large facility might be allocated far in the future. However, there is a benefit to this system. Once it is all agreed upon, the host party will be committed for the long term, not just in the short term as might be the case in the West.

Finally it should be remembered that a working visit to Japan needs to be planned well in advance as the processing of funding request and visa applications takes a minimum of six to nine months.

\section{Language}

More and more young scientists or graduate students arrive in Japan with extensive Japanese language training. This is especially worthwhile for those spending longer periods in Japan and who are in daily contact with more junior people who are often less fluent in English. But not everyone has had the opportunity or the time to study the language before visiting. The most frequently asked question is "What is the useful minimum?"

A French professor who had spent eight years in Japan and spoke Japanese fluently gave me the following advice, "It is going to be very difficult to master the language, so before you go, concentrate instead on learning a few key spoken words of politeness/greetings which are important in daily life." He was right and I strongly recommend it. It makes life easier and presents a good image to the hosts. As it is anywhere, it is a sign of respect for the culture and country. Such words include thank you (arigato) (in various degrees), please (oneigaishimasu), hello, goodbye, a word which is used before starting a meal (itadakimasu), a word during the meal (e.g., delicious [oishiidesu]), a word for after having been treated to a meal (gochisosama), and so on. Visitors who then finally utter one of those words in Japan will undoubtedly be told that they speak Japanese very well-a way of acknowledging their efforts.

Also very important is the attitude when communicating that one wishes to speak in the host language, even if unable. This can be expressed by speaking English very slowly and clearly, avoiding jargon, etc.

\section{Communication is usually indirect, so you often have to look for hidden meaning.}

Once in Japan, visitors should continue weekly lessons in Japanese to facilitate communication with their colleagues. Sometimes, such language courses are conveniently offered through the host institutions.

\section{"Imagine You Are There"}

Undoubtedly someone will pick you up when you arrive in Japan and help you settle in. Even if you and your host called each other by first name elsewhere, the norm in Japan is to call people by their last name followed by -san (i.e., Honda-san) or some ending reflecting rank (i.e., Honda-sensei, typical for professors). This is true even when you are speaking in English. Some Japanese will tell you to switch upon arrival, others will not. It might seem formal to use the last name with -san, but in Japan it is so common that it does not seem formal. The true formality is expressed by other means in the Japanese language.

Even if you have visited Japan for a conference, or pleasure, you will probably notice that the treatment at the laboratory differs from earlier experiences now that you have come to stay for some time. Probably you will be tempted to think that what you are experiencing is the general rule for Japan; for example, in your industrial laboratory everyone wears a uniform, so that is true for all industrial laboratories. Of course there are some overriding cultural patterns, but often what you might experience is due to local customs of the group or reflects the personality of the group leader or professor, just as it would in the West.

You will probably share office space with several people, perhaps even tens of people. In the beginning, it takes some adjustment but you will notice that the people generally speak in much softer voices in order not to disturb their neighbors. Japanese are polite to each other and rarely, if ever, show any strong expressions of disagreement in the workplace. When your colleague or supervisor makes a suggestion, it is typically more than a suggestion in the Western meaning; it is strong recommendation or even an order if the person is your superior. Communication is usually indirect, so you often have to look for hidden meaning. You have to be patient; things are often getting done or being arranged even if it might not appear so. Another big difference with the West is the time given for reflection before expressing one's opinion or the time between a question and an answer in a conversation. Some Westerners feel awkward by the amount of silence or pauses and might think it is due to the language barrier. It is well worth following the rhythm of your hosts.

Foreign scientists have complained about being excluded from meetings or seminars ("I am kept in the dark") and being included ("They are a waste of time; they beat around the bush"). I recommend that you attend occasionally to show your interest and place in the group, even if you do not understand the language. You will understand some of the science despite the language.

The society is extremely structured and organized for the better and for the worse. As everyone knows, the culture is also very group oriented. This is positively reflected when you have a problem; you will be helped and supported by the group to which you belong. At the same time you might be exasperated by conformism that the group behavior produces. Often when you want to do something differently, in a way in which nobody has done before (i.e., no precedence), you will face great difficulties or strong objections, especially if you are young. Socially, you should do your best to adjust. However, if the issue has to do with science, I suggest listening to their objections, but not give in if it hurts the creative process. Obviously, at the frontier of science and technology little precedence is established, so one often has to take a fresh approach to solve a problem.

You can learn from other foreign researchers who are already there. Because of the difference in culture, you will hear strong reactions both positive and negative depending on the host group and the cultural background of the researcher. Associations of foreign scientists and engineers have been formed such as NEST (a network of European researchers) which organizes meetings to discuss experiences and help in the integration process. Such organizations are very useful in that their voices are much stronger in pushing for changes. In the Japanese system, associations $(\mathrm{kai})$ are the best vehicles for expression and reforms. 


\section{Other Issues}

Housing is generally not a problem because the host institutions in Japan typically have some form of housing available at subsidized rates. Many Japanese employees of public institutions or large companies live in such subsidized housing. The quality of this type of housing has improved over the years, but it depends very much on the location. For those bringing their families, the housing situation should be carefully checked with regards to other factors such as access to schools and other essentials.

Schooling is an important issue for older children. 5 The best locations have two options: Japanese schools and international schools. The choice can be difficult to make since the effect on the child will depend on the length of the visit and the child's age. Children attending a Japanese school will learn spoken Japanese quickly. Unfortunately, during this period, the children learn little else since it takes a year or two to acquire a minimum level of speech, writing, and reading to be functional in other courses. It is well-known that while children learn a foreign language easily, they also forget quickly. So for researchers planning to spend many years in Japan, Japanese schools are a very good option, particularly if the children are of elementary school age. Otherwise, children should attend international schools.

There are quite a few international schools in the bigger cities. Typically, they offer quality education in English, and Japanese language training is part of the curriculum. The difficulty for most researchers is the cost of these private schools. The NSF-Japan programs offer educational allowances for children; however, none of the Japanese programs do. If a researcher is not located in one of the major cities, access to such a school can be a problem..$^{5}$ For instance, Tsukuba, a city located an hour north of Tokyo, was built to be an international technopolis, but the city planners never thought of the schooling problem. In response to complaints and demands by visiting foreign scientists, the Tsukuba International School was established without any support from local institutions. Without education allowances from the host institutions, the growth of such schools in academic communities is limited because most researchers cannot afford the full tuition.

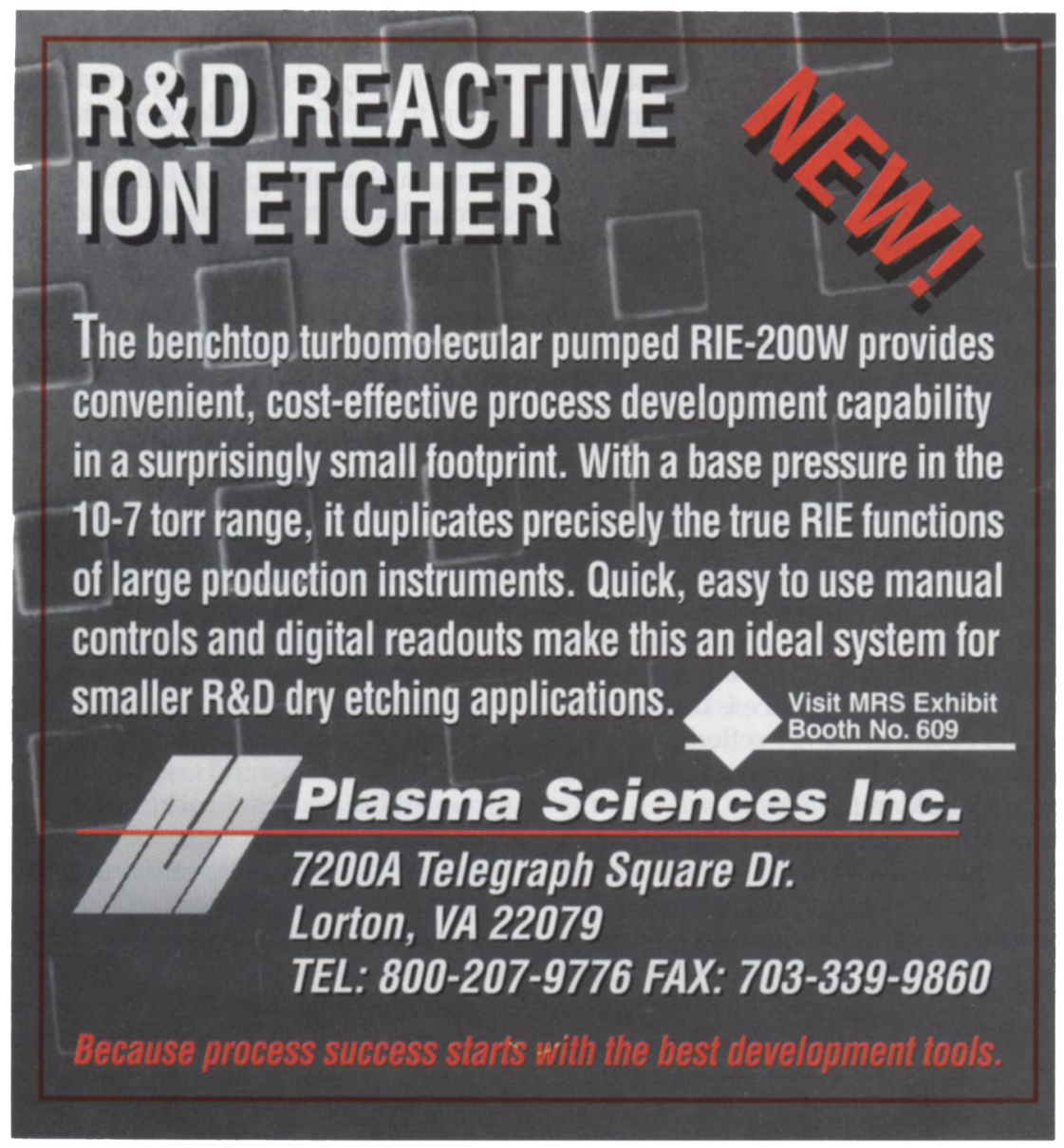

\section{In Conclusion}

The experience of working in Japan can be enriching so long as expectations on both sides are clearly met. While the situation has much improved, the internationalization of the Japanese research environment is still in the making. At the present pace, I believe it will only be another decade before this is completed. Cultural differences will remain, but the understanding of the differences, I hope, will improve. It is important to see the situation as a whole and not to over-react to isolated events or aspects of a foreign culture. There is a balance to each culture.

\section{References}

1. T.W. Ebbesen, Nature 343 (1990) p. 588.

2. D. Normile, Science 261 (1993) p. 1774.

3. D. Swinbanks, Nature 362 (1993) pp. $867-70$.

4. T.W. Eboesen, Butsuri 46 (1991) pp 44-46.

5. D. Swinbanks, Nature 360 (1992) p. 615.

Thomas W. Ebbesen has spent over eight years (of the last 10) in Japan. He is a Research Manager in the Fundamental Research Laboratory of NEC Corporation in Tsukuba. His first experience working in a Japanese laboratory was in 1980 at Tokyo University. He also spent two years at Tsukuba University as a visiting scientist and visiting professor. He is presently on leave from NEC Japan as a visiting scientist at the NEC Research Institute in Princeton, New Jersey.

\section{THE MRS BULLETIN VALUES YOUR OPINION} on matters of interest to the materials community

Write to: Editor, MRS Bulletin

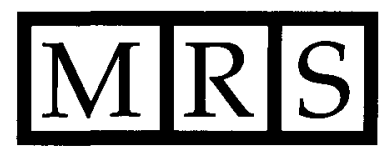

Materials Research Society 9800 McKnight Road Pittsburgh, PA 15237-6006

Phone 412-367-3003 Fax 41 2-367-4373 


\section{Morton's Bulk}

\section{CVD Sillicon Carbide}

Shapes The
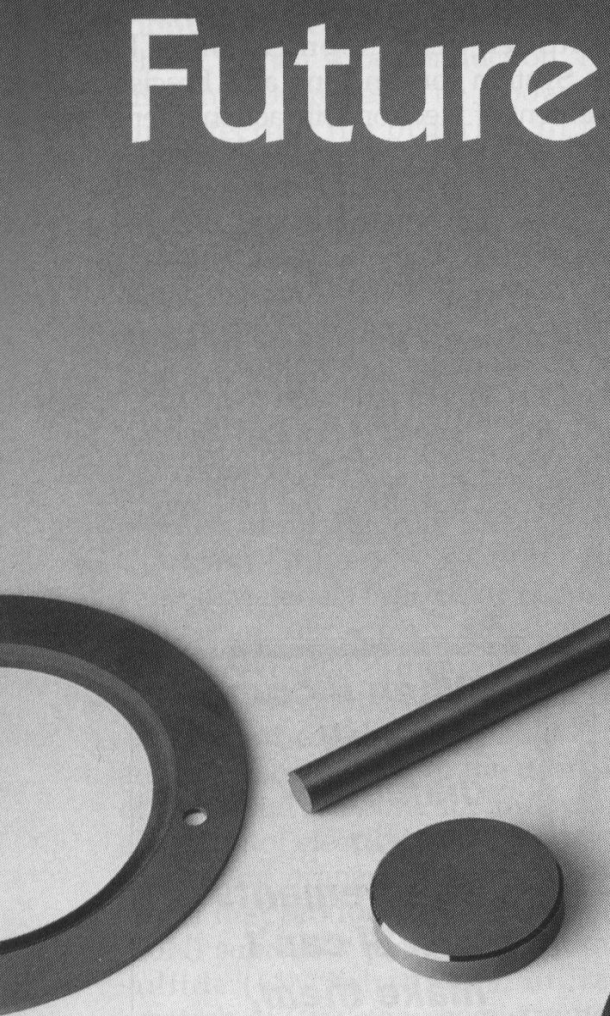

Introducing a new technological breakthrough that now makes it possible to specify cubic (B) SiC components in thicknesses up to one inch and lengths up to 60 inches.

Morton Advanced Materials adds a new dimension to bulk, chemically vapor-deposited beta silicon carbide. A free-standing, monolithic substrate of high purity (99.999\%), CVD Silicon Carbide ${ }^{\text {TM }}$ provides superior chemical resistance, thermal conductivity, stiffness, and polishability ( $<1$ Angstrom RMS). In automotive, optics, information storage media and electronic packasing applications, CVD Silicon Carbide is shaping new ideas. Find out how it can work for you. For more information or a price and delivery quotation for CVD Silicon Carbide, call or write:

Morton International, Inc.

185 New Boston Street, Woburn, MA 01801 800-552-2283 FAX: 617-933-5142

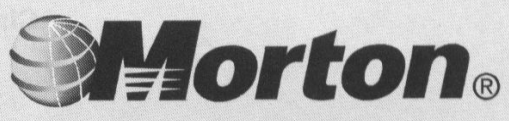

Circle No. 18 on Reader Service Card. 